\title{
Original Research Article \\ Klebsiella Pneumoniae, an Important Uropathogen: Prevalence and Antimicrobial Susceptibility Pattern
}

\author{
Soni P. ${ }^{1}$, Nerurkar A. ${ }^{2}$, Gandhi V. ${ }^{3}$ \\ ${ }^{1}$ Dr. Payal Soni, Tutor, ${ }^{2}$ Dr. Alka Nerurkar, Professor and Head, ${ }^{3}$ Dr. Vicky Gandhi, Assistant Professor; all author are \\ affiliated with Microbiology Department, GMERS Medical College and Hospital, Valsad, Gujarat, India.
}

Corresponding Author: Dr. Payal Soni, Tutor, Microbiology Department, GMERS Medical College and Hospital, Valsad, Gujarat, India. E-mail: drpayalsoni@gmail.com

\begin{abstract}
Introduction: Urinary Tract Infections (UTIs) are amongst the $2^{\text {nd }}$ most common infections after respiratory tract infections in community and healthcare settings (Hospital acquired infections). Klebsiella pneumoniae is the 2nd most common uropathogen after Escherichia coli. Klebsiella is known to have several virulence factors and resistant strains are rising which includes Extended-spectrum Beta-lactamase (ESBL) producing and Carbapenem-resistant strains. Aim of the study is to assess the prevalence of Klebsiella pneumoniae as an Uropathogen and their antimicrobial susceptibility pattern at a tertiary care hospital, Valsad. Material and Methods: Retrospective study is conducted including isolates of Klebsiella pneumoniae from urine samples received in microbiology laboratory at a tertiary care hospital, Valsad, Gujarat, India from January 2018 to December 2018. Processing of samples, isolation and identification of Klebsiella pneumoniae strains with Antimicrobial susceptibility testing were done using standard microbiology techniques. Result: Out of 576 urine samples received in 2018, 209 (36.28\%) showed significant growth. Prevalence of Klebsiella pneumoniae as Uropathogen is $9.72 \%(n=576)$ and their antimicrobial susceptibility pattern shows higher resistance to $\beta$ lactum group (Penicillin, Cephalosporins and Carbapenem). Susceptibility is maximum against Chloramphenicol (87.5\%) followed by Nitrofurantoin (64.29\%), Amikacin (62.5\%), Imipenem (60.71\%), Meropenem (60.71\%), Levofloxacin (57.14\%) and Piperacillin/Tazobactum (57.14\%).Conclusion: Klebsiella pneumoniae remains an important Uropathogen both in community and Hospital acquired infections. Resistance among Klebsiella pneumoniae strains shows upward trends over last few years. Increasing prevalence of ESBL producing and Carbapenem resistant strains creates alarming situation for healthcare system.
\end{abstract}

Key words: Klebsiella pneumoniae, Antimicrobial susceptibility pattern, Uropathogen

\section{Introduction}

Klebsiella species are commensal in human intestines and saprophyte in soil. Klebsiella pneumoniae, an opportunistic pathogen, is responsible for lobar pneumonia, urinary tract infections, meningitis, septicemia and pyogenic infections in community and health care settings [1,2]. Urinary Tract Infections (UTIs) are amongst the $2^{\text {nd }}$ most common infections after respiratory tract infections in community and healthcare settings (Hospital acquired infections), which is responsible for increased morbidity and economic burden to the community. Klebsiella pneumoniae is the 2nd most common uropathogen after Escherichia coli $[3,4]$. Capsular polysaccharides, lipopolysaccharide (LPS) and siderophores are important virulence factor associated with Klebsiella [5]. K. pneumonia strains can

Manuscript received: $10^{\text {th }}$ March 2019

Reviewed: $20^{\text {th }}$ March 2019

Author Corrected: $25^{\text {th }}$ March 2019

Accepted for Publication: $27^{\text {th }}$ March 2019 acquire a variety of $\beta$-lactamase enzymes, which can destroy the chemical structure of $\beta$-lactam antibiotics such as penicillins, cephalosporins and carbapenems, which are most widely used antibiotics [6]. Klebsiella pneumoniae is identified as one of the ESKAPE (Enterococcus faecium, Staphylococcus aureus, Klebsiella pneumoniae, Acinetobacter, Pseudomonas aeruginosa and Enterobacter) pathogens by the Infectious Diseases Society of America (IDSA) in 2004, which are considered as greatest threat, due to the emergence of strains that are resistant to all or most available antibiotics $[2,7]$.

According to Center of Disease Control (CDC), Extended-spectrum Beta-lactamase (ESBL) producing and Carbapenem-resistant Enterobacteriaceae (CRE) are considered as serious and urgent threat for the whole world [8]. 
Based on current scenario, it is of prime importance to know about prevalence of Klebsiella pneumoniae and its antimicrobial susceptibility pattern at local level to deal with it effectively.

Aim of the study is to assess the prevalence of Klebsiella pneumoniae as an Uropathogen and their antimicrobial susceptibility pattern at a tertiary care hospital, Valsad.

\section{Material and Method}

Setting: Tertiary care hospital, Valsad, Gujarat, India

Type of study: Retrospective study

Study duration: January 2018 to December 2018

Inclusion criteria: All samples of urine received in microbiology laboratory in sterile container which shows growth of Klebsiella pneumoniae.

\section{Original Research Article}

Exclusion criteria: All samples of urine which shows growth other than Klebsiella pneumoniae or no growth.

Sample processing: Cultures of urine samples were done on Blood agar (Semi-quantitative method) and MacConkey agar. $10^{5}$ Colony forming unit count is considered as significant growth. Klebsiella pneumoniae strains were identified using standard microbiology techniques [9].

Antimicrobial susceptibility testing of Klebsiella pneumonia strains were performed on Muller Hinton agar using disk diffusion (modified Kirby-Bauer) method as per Clinical laboratory Standard Institute guideline 2018 [10]. Standard strains used were ATCC E. coli 25922, ATCC $S$. aureus 25923 and ATCC $P$. aeruginosa 27853.

Data analysis: Data were entered in excel sheet and analyzed using Microsoft Excel.

\section{Result}

Out of 576 urine samples received in 2018, 209 (36.28\%) showed significant growth.

Table-1: Frequency and percentage positivity of different organisms isolated from urine samples $(\mathbf{n}=\mathbf{2 0 9})$

\begin{tabular}{|c|c|c|c|}
\hline Sr. No. & Organism & Frequency (Out of 209) & Percentage positivity (\%) \\
\hline 1 & Escherichia coli & 83 & 39.71 \\
\hline 2 & Klebsiella pneumoniae & 56 & 26.79 \\
\hline 3 & Enterococcus spp. & 28 & 13.40 \\
\hline 4 & Pseudomonas aeruginosa & 14 & 6.70 \\
\hline 5 & Acinetobacter spp. & 11 & 5.26 \\
\hline 6 & Proteus spp. & 8 & 3.83 \\
\hline 7 & Staphylococcus aureus & 5 & 2.39 \\
\hline 8 & Other & 4 & 1.91 \\
\hline
\end{tabular}

Table-1 shows Escherichia coli as commonly isolated organism followed by Klebsiella pneumoniae and other organisms.

Table-2: Age and sex wise distribution of Klebsiella pneumoniae strains $(\mathbf{n}=56)$

\begin{tabular}{|c|c|c|c|}
\hline Age in years & Male & Female & Total \\
\hline $1-18$ & 6 & 7 & 13 \\
\hline $18-55$ & 8 & 28 & 36 \\
\hline$>55$ & 5 & 2 & 56 \\
\hline Total & 19 & 37 & 7 \\
\hline
\end{tabular}

The chi square statistic is 7.4586 . The p-value is $0.024<0.05$. (Significant difference)

Table-2 shows age and sex wise distribution of Klebsiella pneumoniae strains. Age group 18-55 years is more common followed by age group $1-18$ and $>55$ years.

Females are more affected than male except in age group $>55$ years, where males are more affected. This difference is statistically significant according to chi square test. 
Table-3: Location wise distribution of Klebsiella pneumoniae strains $(\mathrm{n}=56)$

\section{Original Research Article}

\begin{tabular}{|c|c|}
\hline Location & No. \\
\hline Outdoor Patient Department (OPD) & 18 \\
\hline Indoor Patients Department (IPD) & 38 \\
\hline
\end{tabular}

Table-3 shows location wise distribution which shows higher isolation in indoor patients.

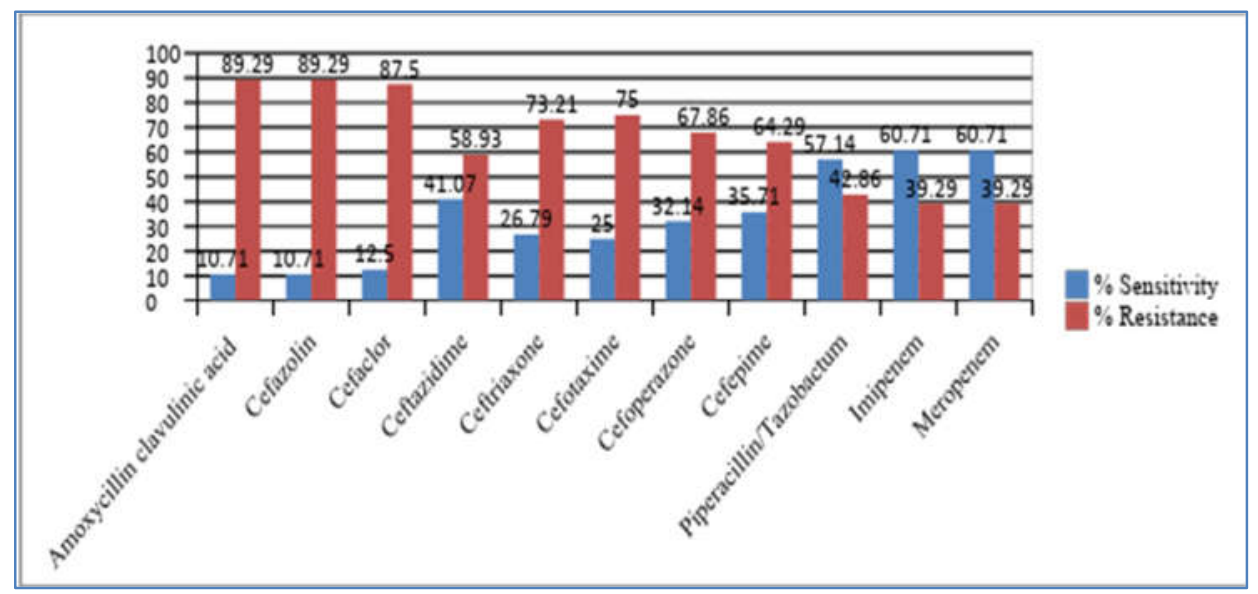

Figure-1: Antimicrobial susceptibility pattern ( $\beta$-lactam group) of Klebsiella pneumoniae $(\mathrm{n}=56)$

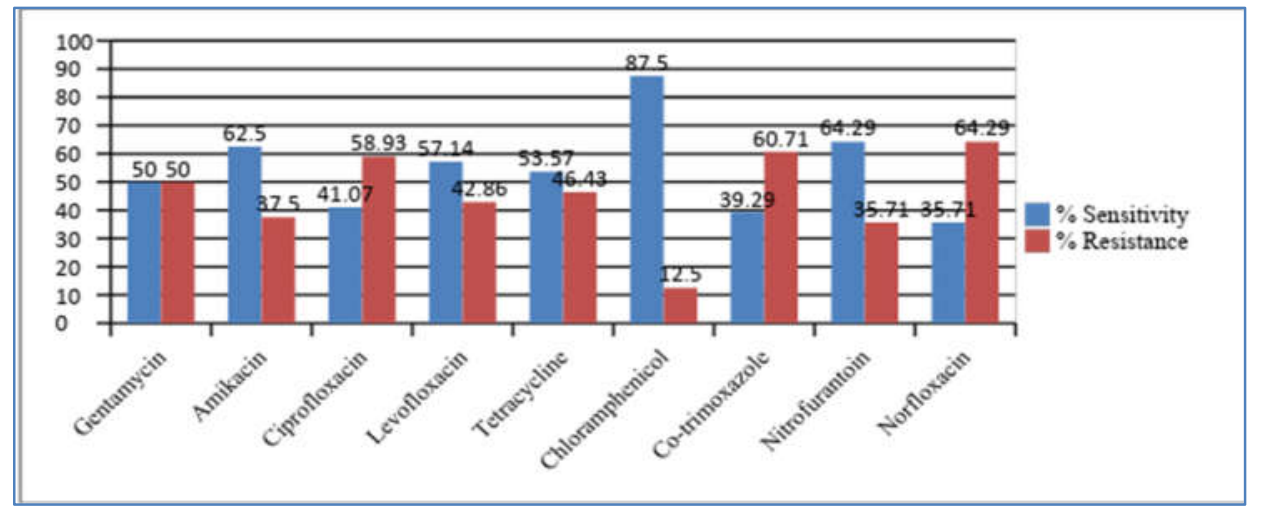

Figure-2: Antimicrobial susceptibility pattern (Other than $\beta$-lactam group) of Klebsiella pneumoniae $(\mathbf{n}=56)$

Figure-1 and 2 shows antimicrobial susceptibility pattern of Klebsiella pneumoniae strains. Susceptibility to Chloramphenicol is $85.5 \%$ followed by Nitrofurantoin (64.29\%), Amikacin (62.5\%), Imipenem (60.71\%) and Meropenem (60.71\%), Piperacillin-Tazobactum (57.14\%) and Levofloxacin (57.14\%). Cephalosporins shows higher resistance.

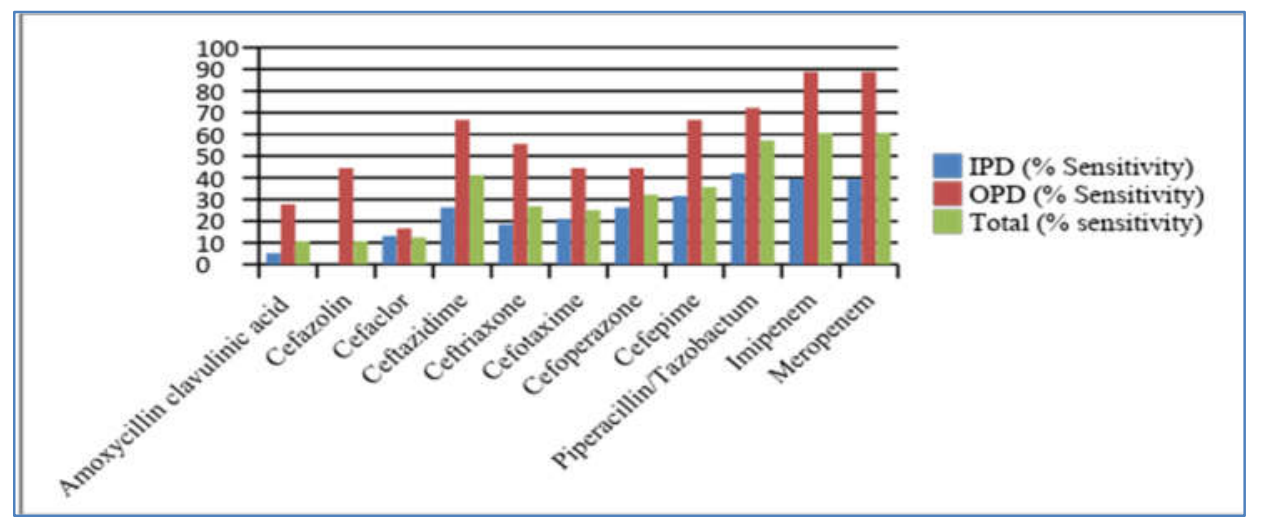

Figure-3: Comparison of \% susceptibility of OPD $(n=18)$, IPD $(n=38)$ and Total $(n=56)$ isolates $(\beta$-lactam group) 


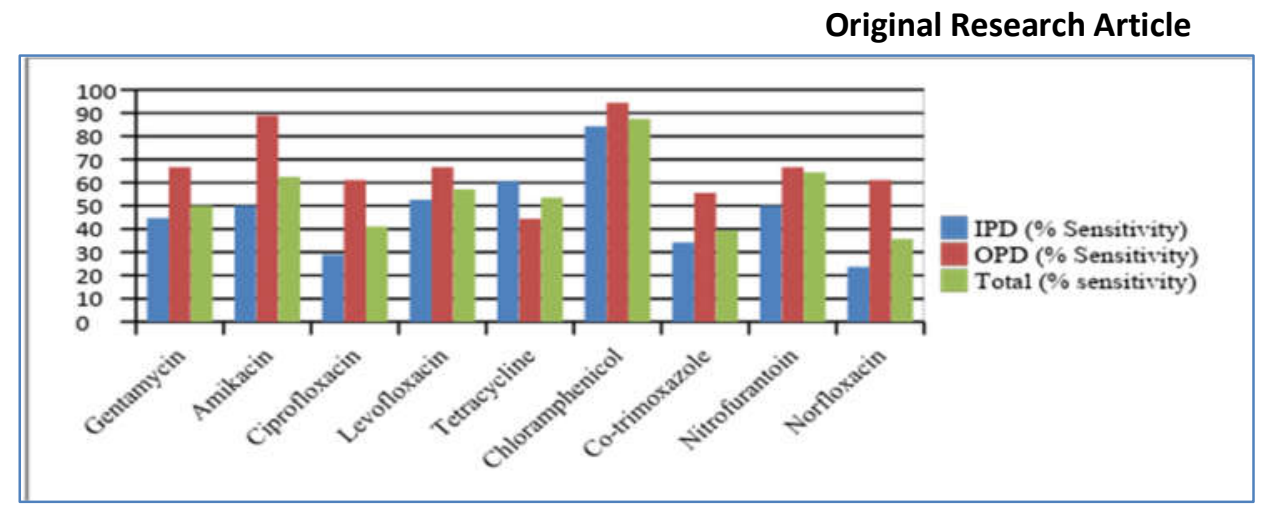

Figure-4: Comparison of \% susceptibility of OPD $(n=18)$, IPD $(n=38)$ and Total $(n=56)$ isolates (Other than $\beta$-lactam group)

Figure-3 and 4 show comparison between susceptibility of OPD, IPD and Total strains. OPD strains show good susceptibility to antimicrobial agent as compare to IPD strains.

\section{Discussion}

Prevalence of Klebsiella pneumonie as Uropathogen is $9.72 \%(\mathrm{n}=576)$ and their antimicrobial susceptibility pattern shows higher resistance to $\beta$-lactam group (Penicillin, Cephalosporins and Carbapenem). Susceptibility is maximum against Chloramphenicol (87.5\%)followed by Nitrofurantoin (64.29\%), Amikacin (62.5\%), Imipenem (60.71\%), Meropenem (60.71\%), Levofloxacin (57.14\%) and Piperacillin/ Tazobactum $(57.14 \%)$.

Table: 1 shows frequency of different organisms isolated from urine samples. Escherichia coli (39.71\%) is commonly isolated bacteria which is comparable with similar study conducted by Vicky Gandhi et al [11] which shows $36.75 \%$ and Harshkumar B. Patel et al [12]. which shows $36.11 \%$ prevalence of the same, followed by Klebsiella pneumoniae (26.79\%) which is higher as compare to similar study conducted in Gujarat by Vicky Gandhi et al [11] which shows $18.21 \%$, Harshkumar B. Patel et al. [12] which shows $18.06 \%$, Parevee Dalalet al [13]. which shows $14.22 \%$, Disha Sharma et al [14]. Which shows 9.04\% and Nilofar R. Sodagar et al [15]. which shows $18.3 \%$ prevalence of Klebsiella pneumoniae. Table: 3 shows location wise distribution of Klebsiella pneumoniae strains. Increased prevalence of Klebsiella pneumonia in Indoor patients may suggest increasing nosocomial infection which creates alarming situation.

Table: 2 shows age and sex wise distribution, which is statistically significant ( $\mathrm{p}$ value: 0.024). Higher prevalence in sexually active females of 18-55 years age group is because of shorter urethra, proximity of urethral meatus to anus and reinfection [1]. In male of age group $>55$ years, prevalence is high due to age related prostatic enlargement which interferes with emptying of bladder [1].
Figure: 1 and 2 shows Antimicrobial Susceptibility pattern of Klebsiella pneumoniaeagainst different antimicrobial agents. $42.86 \%$ were ESBL producer. $39.29 \%$ strains were resistant to Carbapenem. Susceptibility pattern is comparable with similar study conducted in Ahmedabad, Gujarat by Harshkumar et al [12] and Parevee Dalal et al [13].

In present study, Susceptibility to Imipenem (60.71\%), Levofloxacin (57.14\%) and Cephalosporins (10-30\%) is lower as compare to similar study conducted in Valsad by Niraj kumar Biswas et al [16] in 2014 (Imipenem 91.82\%, Levofloxacin $66.70 \%$ and Cephalosporins $40-$ $60 \%$ ) and Vicky Gandhi et al [11] in 2016 (Imipenem $87 \%$, Levofloxacin $60 \%$ and Cephalosporins 20-40\%). Study conducted by Shankar Srinivasan et al [17] in 2014 at Mumbai shows Susceptibility of 89-90\% for Carbapenems and Levofloxacin. Susceptibility to Nitrofurantoin is $64.29 \%$ which is comparable with study conducted at Udaipur by Ritu Bhatnagar et al [18] (68.57\%).

This scenario suggests growing resistance among bacterial strains against commonly used flouroquinolones and cephalosporins as well as last resort drug, carbapenems. Resistances against commonly used antimicrobials create selective pressure on last resort drug. Selective pressure along with over the counter drugs, lack of regulations and unnecessary uses of antimicrobials are important factors responsible for increased resistance in developing country like India [19].Change in susceptibility pattern suggests increasing trend of resistance among Klebsiella strains. Antimicrobial policy should be made according to the susceptibility pattern. Hospital infection control measures should be followed stringently to control this situation. 
Klebsiella pneumoniae is important nosocomial infections with higher chances of infection in immunocompromised patients, patients on mechanical ventilation, intravenous catheters, urinary catheters or on long course of immunosuppressant drugs [20]. Figure: 3 and 4 shows Comparison of \% Susceptibility of OPD $(n=18)$, IPD $(n=38)$ and Total $(n=56)$ isolates against different antimicrobial agents. Strains isolated from OPD patients shows higher Susceptibility even to Cephalosporins as compare to strains isolated from IPD strains. This may suggests increasing prevalence of nosocomial infections by resistant strains. Community acquired strains still have good Susceptibility against commonly used antimicrobials. Nitrofurantoin, an age old oral drug, can be used as an alternative for OPD patients.

Klebsiella pneumonie can acquire resistance against Carbapenem and other antimicrobial by various mechanisms which includes enzymatic inactivation, target site modification, efflux pump etc. Carbapenamase enzymes have significant impact on usages of antimicrobials in gram negative infections. These resistances are plasmid coded and can be transferred to other bacteria also, which increases chance of resistance among other bacteria in health care settings [21].

Further classification of Carbapenem resistant Klebsiella pneumoniae strains can be done based on Ambler classification [22]. Phenotypic and Molecular methods can detect different enzyme and its corresponding genes, which can help to evaluate prevalent resistant strains. Due to lack of testing facility at present study setup, it remains limitation for this study.

\section{Conclusion}

In community and Hospital acquired infections, Klebsiella pneumoniae remains an important Uropathogen. Resistance among Klebsiella pneumoniae strains shows upward trends over last few years. Increasing prevalence of ESBL producing and Carbapenem resistant strains creates alarming situation for healthcare system. Phenotypic and Molecular level gene analysis should be done to identify prevalence of resistant strains. Active surveillance system and stringent hospital infection control measures should be implemented to control further rise.

This study provides prevalence and Antimicrobial susceptibility pattern of Klebsiella pneumoniae as Uropathogen at local level to create antibiogram for Hospital infection control policy to deal with it effectively.

\section{Original Research Article}

Contribution of authors: Bacterial identification and reporting of all samples were done by Dr. Payal Soni and Dr. Vicky Gandhi under the guidance of Dr. Alka Nerurkar. All authors have contributed in manuscript writing.

Funding: Nil; Conflict of Interest: None initiated Permission from IRB: Yes

\section{References:}

1. Apurba Shankar Sastry, Sandhya Bhat K. Essentials of Medical Microbiology. New Delhi: Jaypee Brothers Medical Publishers (P) Ltd; 2016.

2. Wyres KL, Holt KE. Klebsiella pneumoniae as a key trafficker of drug resistance genes from environmental to clinically important bacteria. Curr Opin Microbiol. 2018 Oct;45:131-139. doi: 10.1016/j.mib.2018.04.004. Epub 2018 May 1.

3. Gatermann SG. Bacterial infections of the urinary tract. In: Borriello P, Murray PR, Funke G, editors. Topley\&Wilson's microbiology \& microbial infections, London: Hodder Arnold Publishers 10 $0^{\text {th }}$ d, 2007; vol III:p 671-683.

4. Anila Varghese, Shareen George, Radha kuttyamma Gopalakrishnan, Anna Mathew. Antibiotic susceptibility pattern of Klebsiella pneumoniae isolated from cases of urinary tract infection in a tertiary care setup. J. Evolution Med. Dent. Sci 2016 Apr. 11, 5(29): 14701474.

5. Shilpa K, Ruby Thomas and AllavarapuRamyashree. Isolation and Antimicrobial Susceptibility pattern of Klebsiella pneumoniae from sputum samples, International Journal of Biomedical and Advance Research, 2016, 7(02):053-057.

6. SirijanSantajit and NitayaIndrawattana. Mechanisms of Antimicrobial Resistance in ESKAPE Pathogens. Bio Med Research International, 2016, Article ID 2475067, http://dx.doi.org/10.1155/2016/2475067.

7. Brews C. Infectious Diseases Society of America. Bad Bugs, No Drugs. As Antibiotic Discovery Stagnates. A Public Health. (2004).

8. Biggest threats and data [Internet]. 2019 [updated 2018 Sep 10; cited 2019 Apr 15]. Available from: https: //www.cdc.gov/drugresistance/biggest_threats.html

9. Collee JG, Miles RS, Watt B. Tests for the identification of bacteria. In: Collee JG, Fraser AG, Marmion BP, Simmons A, editors. Mackie and Mc 
Cartney Practical Medical Microbiology. 14th ed. Edinburgh: Churchill Livingstone; 1996. p. 131-150.

10. M100, Performance Standards for Antimicrobial Susceptibility Testing. $28^{\text {th }}$ edition. Pennsylvania, USA: Clinical Laboratory Standard Institute; 2018.

11. Vicky Gandhi, Mehul Patel, Alka Nerurkar. Bacteriological profile and its antibiotic susceptibility in patients with Urinary Tract Infection at Tertiary Care Hospital, Valsad, Gujarat. International Journal of Medical Microbiology and Tropical Diseases, AprilJune, 2017;3(2):57-60.

12. Harshkumar B. Patel, Sumeeta T. Soni, Aroor Bhagyalaxmi, Neev M. Patel. Causative agents of urinary tract infections and their antimicrobial susceptibility patterns at a referral center in Western India: An audit to help clinicians prevent antibiotic misuse. J Family Med Prim Care 2019;8:154-9.

13. PareveeDalal, JayshriPethani, HetalSida, Hiral Shah. Microbiological profile of urinary tract infection in a tertiary care hospital, Journal of Research in Medical and Dental Science. Jul-Sep 2016: 4 (3): 204-209.

14. Disha Sharma, Yagnesh Pandya, Suman Singh. Identification, Antimicrobial Resistance Patterns and Factors Associated With Urinary Tract Infections In A Tertiary Care Teaching Hospital of Rural Gujarat-India. Natl J Integr Res Med 2017; 8(5):13-18.

15. Nilofar R. Sodagar, Dr. Sunil S. Trivedi. Microbiological profile and antibiotic susceptibility pattern of Uropathogens in Anand district of Gujarat, India. Journal of Biomedical and Pharmaceutical Research. Jul-Aug 2015;4(4): 51-59.

\section{Original Research Article}

16. Biswas NK, Patel PH, Ahir HR, Kothadiya PH, Nerurkar AB. Bacteriological Profile Of Urinary Tract Infection And Its Antibiotic Susceptibility At Tertiary Care Hospital, Valsad, Gujarat, India. Journal of Pharmaceutical and Biomedical Science.2014;04(09): 751-754.

17. Shankar Shrinivasan, Jovita Saldanha, Suhas Abhyankar, Nikunj Modi, Aakanksha Patil, Arvind M Vartak. Antibiotic Susceptibility pattern of Klebsiella species in burn wounds at Bai Jerbai Wadia hospital for children, Mumbai, India-a 21 year study. Int J Burn Trauma 2017;7(5):64-71.

18. Ritu Bhatnagar, PayalSoni, Sanjeev Kumar, S.K. Mehra, Aruna Solanki, Jyoti Tomar. Study of Nitrofurantoin susceptibility among uropathogenic bacterial isolates at a tertiary care hospital, Udaipur, Rajasthan. World Journal of Pharmaceutical Research. 2015; 4(5): 2124-2132.

19. Sojib Bin Zaman, Muhammed Awlad Hussain, Rachel Nye, Varshil Mehta, Kazi Taib Mamun, Naznin Hossain. A Review on Antibiotic Resistance: Alarm Bells are Ringing. Cureus 9(6): e1403. DOI 10.7759/ cureus. 1403.

20. Woldu MA. Klebsiella pneumoniae and Its Growing Concern in Healthcare Settings. Clin Exp Pharmacol. 2016; 6: 199. doi:10.4172/2161-1459.1000199.

21. Francis S. Codjoe, Eric S. Donkor. Carbapenem Resistance: A Review. Med Sci (Basel). 2018 Mar; 6(1): 1. doi: 10.3390/medsci6010001.

22. Karen Bush, George A. Jacoby. Updated Functional Classification of $\beta$-Lactamases. Antimicrobial agents and chemotherapy. Mar. 2010; 54(3): p. 969-976

\section{How to cite this article?}

Soni P, Nerurkar A, Gandhi V. Klebsiella Pneumoniae, an Important Uropathogen: Prevalence and Antimicrobial Susceptibility Pattern. Trop J Path Micro 2019;5(3):171-176.doi:10.17511/jopm.2019.i03.10. 